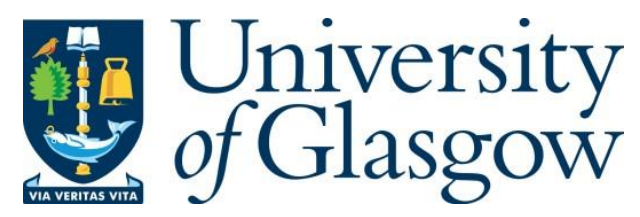

Pea, A., Jamieson, N. B. and Braconi, C. (2021) Biology and clinical application of regulatory RNAs in hepatocellular carcinoma. Hepatology, 73(S1), pp. 38-48. (doi: 10.1002/hep.31225).

There may be differences between this version and the published version. You are advised to consult the publisher's version if you wish to cite from it.

This is the peer reviewed version of the following article:

Pea, A., Jamieson, N. B. and Braconi, C. (2021) Biology and clinical application of regulatory RNAs in hepatocellular carcinoma. Hepatology, 73(S1), pp. 38-48, which has been published in final form at 10.1002/hep.31225. This article may be used for non-commercial purposes in accordance with Wiley Terms and Conditions for Self-Archiving.

http://eprints.gla.ac.uk/212482/

Deposited on: 27 March 2020

Enlighten - Research publications by members of the University of Glasgow http://eprints.gla.ac.uk 
DR. ANTONIO PEA (Orcid ID : 0000-0002-0509-6756)

Article type : Review

\section{Biology and clinical application of regulatory RNAs in hepatocellular carcinoma}

Antonio Pea, ${ }^{1,2}$ Nigel B Jamieson ${ }^{1,2}$, Chiara Braconi ${ }^{1,3}{ }^{*}$

${ }^{1}$ The Institute of Cancer Sciences, University of Glasgow, Glasgow, UK

${ }^{2}$ West of Scotland Pancreatic Unit, Glasgow Royal Infirmary, Glasgow, UK.

${ }^{3}$ Beatson West of Scotland Cancer Centre, Glasgow, UK

"Corresponding and lead author: Chiara Braconi, The Institute of Cancer Sciences, University of Glasgow, Glasgow, UK, G61 1QH. Phone: +44(0) 141330 3278. Fax: 01419426521 Email:

chiara.braconi@glasgow.ac.uk

Keywords: microRNA, HCC, biomarker, long ncRNA.

\section{Abstract}

The majority of the human genome comprises of DNA genes that are translated into RNAs but not into proteins. These RNA molecules are named non-coding RNAs (ncRNA). While in the past it was thought that ncRNAs would be redundant without relevant functions, it is now well established that ncRNAs identify a class of regulatory molecules that finely tune cell homeostasis and are deregulated in disease states, including Hepatocellular Carcinoma (HCC). Of note, the number of ncRNAs within a cell increases progressively with the complexity of the species indicating their essential role in the maintenance of regulatory networks that impact the intricacy of the organism. ncRNAs have been demonstrated to mediate HCC development and progression by affecting intrinsic cancer cell signaling and cross talk between malignant cells and the microenvironment. They hold promise as clinical biomarkers, but further evidence is warranted prior to translation and integration within clinical practice.

This article has been accepted for publication and undergone full peer review but has not been through the copyediting, typesetting, pagination and proofreading process, which may lead to differences between this version and the Version of Record. Please cite this article as doi: $\underline{10.1002 / \mathrm{HEP} .31225}$

This article is protected by copyright. All rights reserved 


\section{Introduction}

Over recent years considerable progress has been made in characterizing the molecular alterations underlying the development of $\mathrm{HCC}(1)$. Numerous post-transcriptional RNA modifications regulating multiple gene expression processes have been discovered as a result of advances in molecular biology and detection methodologies. While most modifications occur in the "classical" mRNA and tRNA, several classes of functional RNA that possess no or low coding potential have been identified(2,3). Less than $2 \%$ of the genome encodes for proteins but three-quarters are transcribed in non-coding RNAs (ncRNA) suggesting the existence of an intricate RNA-based network with regulatory functions. NcRNA has been divided in different classes according to molecular size: 1) small RNAs are usually 20-30 nucleotides long being classified into 3 major types in animals: microRNA (miRNA), siRNA and PIWI-interacting RNA (piRNA), 2) long non-coding RNAs (lncRNAs) being > 200 nucleotides.

\section{MicroRNAs}

MiRNAs represent the dominant class of small RNAs, regulating protein-coding genes through translational repression and mRNA degradation(4). Regulation of miRNAs occurs at multiple levels of miRNA biogenesis. Genomic sequences for miRNAs genes are transcribed to primary miRNAs by RNA polymerase II in the nucleus under the positive or negative regulation of transcription factors and epigenetic mechanisms. Pri-miRNAs are then processed by the nuclear RNase III Drosha and by the cytoplasmatic Rnase III Dicer to generate a miRNA complex with two strands. While one strand is depredated, the other is bound onto AGO protein to form an effector complex called RNA-induced silencing complex(RISC)(4). While the majority of evidence suggests that miRNAs bind to the 3' UnTranslated Region (3'-UTR), crosslinking and immunoprecipitation experiments have recently shown that translational inhibition can also occur through the binding of miRNA within the coding region and 5'-UTR(5). MiRNA deregulation happens through several mechanisms in HCC, including chromosomal alterations, promoter methylation and aberrant expression of transcription factors. Recently, mutation of DICER1 has been identified in

$2.3 \%$ of sporadic HCC, that are characterized by a decreased expression of mature miRNAs compared to wild type tumors(6). Taken together, these findings suggest that alterations in the miRNA-processing machinery could potentially influence HCC development(7), underlining an important regulatory role of ncRNAs for the fine-tuning of cell homeostasis.

\section{MiRNAs and HCC Initiation}

The aberrant expression of ncRNAs has been observed at different stages of liver disease and ultimately in HCC. MiR-122 is the most abundant miRNA in the liver, accounting for more than $70 \%$ of the hepatocyte miRNome. Integral to various metabolic functions including fatty-acid metabolism(8), cell differentiation and maintenance of the adult hepatocytes phenotype, miR-122 plays a critical role in promoting 
inflammation-mediated hepatocarcinogenesis, as miR-122 deficient mice develop steatohepatitis, fibrosis and spontaneous liver tumors(9). Different clusters of miRNAs have been identified in association with different etiologies, as pathways involved in regulation of immune response, cell cycle and metabolisms are activated by hepatite $\mathrm{C}$ virus (HCV)-associated miRNAs, whereas pathways regulating cell death, DNA damage and signal transduction are preferentially activated during hepatite B virus (HBV) infection. However, common miRNAs are necessary for the progression from chronic infection to HCC, independent from the predisposing condition(10). Biopsies from patients with Non-Alcoholic Heapatosteatosis (NASH) also showed a specific pattern of 23 deregulated miRNAs(11). Specific miRNAaccording to the different pathogens therefore deliver a synergistic action through impacting on multiple self-sustaining cellular processes, leading to a global impairment of the cellular networks that ultimately drive malignant transformation. The mechiansms by which chronic inflammation, HBV, HCV and NAFLD promote cancer initiation through miRNAs deregulation are detailed in the supplementary materials.

\section{MiRNAs and HCC Progression}

Even though miRNA impairment is an early event in hepatocarcinogenesis, there is evidence for a sequential global miRNA downregulation occurring in the progression from early to metastatic tumors(12)[Figure 1]. For example, miR-122 downregulation promotes the activation of miRNAs/mRNAs usually expressed in embryonic liver and drives a more aggressive phenotype associated with poor clinical outcome(13). Using a mathematical modelling approach, Gerard et al showed how miRNAs interact with protein partners to generate a regulatory network that sustains HCC progression(14) and that includes Let7b, CTNNB1, LIN28B, SOX9, P53 and MYC. The deregulation of this gene regulatory network was associated with HCCs characterized by poor differentiation and prognosis. Such evidence suggest that, as miRNA impairment is an early event in hepatocarcinogenesis, there is a sequential global miRNA downregulation from normal liver tissue to HCC and subsequently during metastases.

Growing evidence is supporting an important role for miRNAs to regulate the cross-talk between cancer cells and microenvironment. MiR-210 is known to respond to hypoxic stress in cancer; in HCC, miR-210 sustains HCC growth in hypoxic conditions via regulation of the vacuole-membrane-protein (VMP)1 and the tissue inhibitor of metalloproteinases (TIMP)2(15,16). Re-organization of the matrixome is also mediated by miR-29b that can downregulate matrix-metaloproteinases (MMP)2, while having also an effect on epithelial cell apoptosis(17). miR-148a affects fibrosis and regulates the plasticity of hepatocytes, while miR-125b and miR-100 promote venous invasion which favors recurrence and metastatisation in HCC $(18,19)$. The onset of distant metastases is a late event in HCC and is nurtured by the transition of cancer cells from an epithelial to a mesenchymal phenotype (ECM). miR-200, miR-139, miR-124, and 
miR-101 remodel the cytoskeleton by acting on the Rho/RoCK signaling, which results in an increased ability of HCC cells to migrate and invade(20-22). EMT is then sustained by paracrine signaling originating from other microenvironment components including cancer-associate-fibroblasts (CAF). Recent work shows that CAFs are recruited in the metastatic niche by the release of miR-1247-containing exosomes from HCC cells(23). In turn, CAFs create a vicious loop by releasing miRNAs (i.e. miR-335), sustaining HCC cell proliferation(24).

MiRNAs are also known to govern the interplay between cancer cells and immune cells. For example, loss of miR-34 can promote immune-escape by altering the profile of cytokines that recruit and activate Tregulatory cells (Treg)(25). Cytokines are also central to a miRNA-mediated negative feedback loop that occurs between HCC cells and tumour associated macrophages (TAM). Lack of miR28-5-p in HCC leads to increased interleukin-34 that recruits TAMs, which in turn inhibit miR28-5 via TGF-B and IL-6; the latter recognized as a promoter of HCC progression through its action on the HCC stem-cells niche(26). Stemness features are then nourished by the crosstalk with tumor-associated neutrophils (TANs) and is mediated by the effect of TAN-derived miR301-3p on HCC cells(27).

\section{miRNAs as clinical molecular biomarkers}

Novel diagnostic tools are required to detect HCC at an early stage as well as serve as prognostic and predictive biomarkers to enhance clinical decision-making [Figure 2].

MiRNAs actively contribute to the development of HCC, and aberrant miRNA expression patterns distinguish HCC from adjacent normal and cirrhotic tissues $(28,29)$. MiRNAs can be released into the circulation 1) passively as free RNAs due to cell death, 2) associated to lipoproteins and RNA binding proteins, or 3) secreted into extracellular vesicles(30). In all cases, circulating miRNAs are present in the circulation in a highly stable form protected from RNAses. Several single circulating miRNAs have been suggested as diagnostic biomarkers, yet to date have demonstrated limited diagnostic accuracy.

Combinations of multiple miRNAs have been demonstrated to increase specificity and diagnostic accuracy for HCC; however, most studies are limited by small sample size and lack of independent validation. Among those studies that identified, and independently validated, panels of miRNA with diagnostic potential, Li et al. evaluated 13 miRNAs associated with HCC identifying miR-25, miR-375, and let-7f to clearly segregate HCC cases from healthy controls $(\mathrm{AUC}=99.9)(31)$. Zhou et al. profiled miRNAs expression in 934 patients and identified a dysregulated miRNA panel composed of miR-122, miR-192, miR-21, miR-223, miR-26a, miR-27a and miR-801, capable of differentiating HCC from healthy controls $(\mathrm{AUC}=0.941)$, chronic hepatitis B $(\mathrm{AUC}=0.842)$, and cirrhosis $(\mathrm{AUC}=0.884)$, independent of HCC stage(32). However, miR-1228 expression was used as a stable endogenous control for normalization, while it is known to promote HCC and being present at higher levels in the plasma of patients with BuddChiari syndrome and $\mathrm{HCC}(33)$; thus making these data difficult to reproduce. 
Lin et al. recently studied a cohort of 1416 Chinese patients with liver diseases, including a nested casecontrol cohort. They identified a panel of 7 miRNAs (miR-29a, miR-29c, miR-133a, miR-143, miR-145, miR-192, and miR-505), which distinguished patients with HCC from individuals with chronic hepatitis B, HBV-induced liver cirrhosis, and healthy controls(34). While promising, further validation is warranted before this panel can be considered for clinical application. As this study comprised mainly of HBVpositive patients, how these data can be translated to HCC secondary to other etiologies requires investigation, given that at least tissue miRNA expression is dependent on the causative factor. Overall, studies of circulating miRNAs as diagnostic biomarkers have not shown high reproducibility, and this may be related, at least partly, to the modulation of circulating miRNAs induced by several pathological conditions including inflammation, sepsis, cardiological and immunological disease. It is likely that their value will be better served within multiplex-assays where miRNA deregulation is paired to other components, such as circulating tumor DNA that enhances specificity.

Neoplastic progression with the development of distant metastases is the primary cause of the high mortality of patients with HCC. Reduced miR-26 tissue expression was shown to be a significant prognostic marker in a large cohort of clinically annotated HCC tissues; it associates with a transcriptomic inflammatory signature exhibiting negative prognostic value after curative surgery, but positive predictive value for response to adjuvant interferon(35). Based on these findings, a prospective clinical trial was launched to inform adjuvant treatment (with Interferon) according to miR-26 expression levels (NCT01681446), with results presently awaited. Various authors have analyzed miRNA expression and associated clinical data from large publicly available HCC datasets, including the TCGA and NCBI Gene Expression Omnibus(36,37), observing various prognostic miRNA signatures. Variability likely reflects the vast heterogeneity among HCC patients in terms of the pathogenic factors and etiologies involved. Tumor progression is known to rely not solely on intrinsic epithelial features, but on the interaction with several cell types that contribute to a favorable soil for tumor growth; given miRNAs play a key role in tuning the crosstalk with the microenvironment, it is likely that they represent promising factors to assess patients' prognosis. However, in order to overcome lack of reproducibility amongst studies, large cohorts are required within the framework of prospective randomized trials.

MicroRNAs have been shown to affect key survival and cell death signaling pathways, determining the cellular stress response induced by drug treatment(38). It is now known that drug response in patients is mediated by a plethora of factors extending beyond tumor to include network signaling within the microenvironment and the host immune response. In addition, patients' comorbidities represent a key factor that impacts fitness, drug metabolism and tolerability, as well as drug response. Thus, the capacity of circulating miRNAs to reflect the overall homeostasis of the organism provides an additional level of complexity with potential to enhance prediction of drug response (especially to non-targeted therapies) and identify suitable patients for treatment. A recent study analysed multiple HCC cell lines and identified 3 
robust transcriptomic subgroups, each characterized by a peculiar differentiation state and by different miRNA profiles. However, when response to different therapeutic agents was evaluated, miRNA expression showed a lower predictive ability than mRNA expression(39), likely reflecting a lack of detail regarding microenvironment and cell-to-cell interaction in this model.

Currently, therapeutic options for advanced HCC include multikinase-inhibitors such as sorafenib and regorafenib. Fornari et al identified circulating miR-221 as a mediator of sorafenib resistance in vitro and in vivo, which was confirmed in small retrospective cohorts of HCC patients(40) while serum miR-181a-5p was recently found to be independently associated with disease control following therapy(41). While prospective validation is required in larger cohorts, these findings highlight how an integrated approach involving interrogation of biological processes and translational analyses hold promise for the identification of predictive biomarkers. The introduction of novel technologies, such as quantitative digital PCR, may define a cut-off value for miRNA expression that can be used prospectively by enabling counting of circulating RNA molecules per volume of fluid. It is expected that the introduction of these technologies within ancillary translational analyses of prospective randomized clinical trials will help to clarify the predictive role of miRNAs in HCC. Such an example is provided by the translational study within the RESORCE trial, which provided evidence for regorafenib treatment in HCC patients(42). Teufel et al reported the clinical value of a set of miRNAs (miR-15b, miR-107, miR-320b, miR-122, miR-374b, miR200a, miR-30a, miR-125b, miR-645) in identifying survival benefit from regorafenib(43). Given the nature of the trial (prospective, randomized, with treatment and control arm), these findings represent the most robust data to support the clinical utility of miRNAs to date(43). However, in order to progress prospectively, a quantitative assessment of RNA molecules is necessary to identify a robust threshold applicable on a patient-per-patient basis. Given that the greatest challenge currently faced in HCC treatment is the identification of patients who derive a long-term benefit from check point inhibitors(44,45), it would be interesting to investigate the role of ncRNAs in predicting immunotherapy response. While no data exist for HCC, a combination of circulating miRNAs and PD-L1 expression defines a niche of lung cancer patients who benefit from immunotherapy(46). Similarly, a panel of aberrant miRNAs was recently shown to be involved in the development of resistance to immunotherapy in melanoma patients(47). Additional evidence suggest that ncRNAs may drive lymphocyte differentiation and that non-coding regions represent the major source of targetable tumor-specific antigens $(48,49)$. Taken together, these findings suggest that aberrantly expressed ncRNA might have a critical role in driving tumor immunogenicity and may potentially serve as predictors of immunotherapy response in HCC.

\section{Long non-coding RNAs}

LncRNAs comprise various RNA species ranging from $200 \mathrm{nt}$ to $\sim 100 \mathrm{~kb}$ in length. They function as ligands for proteins or form complexes through base-pairing interaction with specific RNA and DNA sites. 
Expression of lncRNAs is more tissue specific than miRNAs, and distinct expression patterns exist across different stages of tissue differentiation, indicating different regulatory roles in diverse cellular contexts(50). Nuclear lncRNAs promote transcriptional modulation of targeted genes by recruiting and guiding chromatin modifiers to specific genomic loci. Among these, the polycomb-group-complex 2 (PRC2) is frequently recruited by lncRNAs in HCC mediating gene silencing by establishing a repressive chromatin status. Cytoplasmatic lncRNAs appear to modulate mRNA stability and regulate translational and post-translational modifications, by directly targeting mRNA or by acting as "endogenous competing RNA" that compete with protein-coding genes for for the binding site of regulatory miRNAs, resulting in decreased miRNA activity and detection(51).

\section{LncRNAs and carcinogenesis}

Depending on HCC etiology, different lncRNAs have been demonstrated to contribute to HCC initiation. In HBV-related HCC, the lncRNA HOTAIR is overexpressed and interacts with the PCR2 complex to control chromatin remodeling. Different mechanisms have been postulated to explain the interactions between HOTAIR and the PCR2 subunits, which are mediated by either protein complexes (i.e. SUZ12 and EZH2) or RNAs (i.e.miR-218)(52).

The oncogenic lncRNA HEIH interacts with EZH2 to deregulate the PRC2 complex and promote epigenetic silencing of tumor suppressor genes(53). HBx can induce aberrant expression of lncRNAs with oncogenic function, such as DBH-AS1 and UCA1. DBH-AS1 promotes HCC cell proliferation and tumor growth by suppressing p53 and activating MAPK signaling, while UCA1 suppresses p27 through histone methylation on its promoter(54). In addition, UCA1 reduces expression of the tumor suppressor miR$216 \mathrm{~b}(55)$. The HBV genome can integrate into host DNA to form a hybrid lncRNA transcript called HBxLINE1, detectable in $23 \%$ of $\mathrm{HBV}$ associated HCC, it functions as a molecular sponge binding and sequestering miR-122(56,57). Finally, Hbx can downregulate lncRNAs with tumor suppressive function, such as Dreh, which combines with and alters the structure of the mesenchymal protein vimentin(58). Profiling data on human HCC identified Linc01419 in early stage disease, and AK021443 and AF070632 as lncRNA specific for advanced stage $\mathrm{HCC}(59)$.

\section{LncRNAs prognostic role and cancer progression}

The lncRNA DANCR is over-expressed in cells with stemness features and is clinically associated with HCC recurrence and poor prognosis. Functional analyses revealed that DANCR promotes metastatic progression by associating with CTNNB1 mRNA thereby blocking the repressing effect that miR-214, miR-320a, and miR-199a have on CTNNB1(60). The lncRNA UFC1 interacts with the RNA binding protein HuR preventing $\beta$-catenin degradation and increasing cytoplasmatic levels(61). The ultraconserved ncRNA uc.158- was shown to be downstream of the wnt signaling activation that occurs in malignant 
transformation, providing the basis for using this lncRNA as biomarker for the targeted use of wnt inhibitors(62). Expression of the lncRNA Highly Up-regulated in Liver Cancer (HULC), is associated with intrahepatic metastases, HCC recurrence, and shorter postoperative survival, promoting progression through numerous oncogenic mechanisms. HULC acts as a scaffold for the phosphorylation of YB-1 that accelerates the translation of oncogenic mRNAs, as cyclin D1, cyclin E1, and MMP11(63). In addition, HULC targets MiR-200a-3p resulting in an increased expression of EMT markers(64), it promotes neoangiogenesis by targeting the tumor angiogenesis-associated factors SPHK1(65) and contributes to the abnormal lipid metabolism of hepatoma cells(66). Both lncRNAs Zeb1-AS1 and ZFAS1 are frequently overexpressed in HCC and are clinically associated with the development of metastases and reduced recurrence free survival. They promote neoplastic progression by interacting with the transcription factor ZEB1, a direct repressor of E-cadherin(67,68). ZFAS1 promotes the development of intrahepatic and extrahepatic metastasis by binding miR-150 and abrogating its tumor-suppressive function on ZEB1 and the matrix metalloproteinases(68).

There is evidence that LncRNAs are differently expressed in neoplastic vein thrombus, compared to the matching primary, with ICAM-1-related (ICR) and the lncRNA-activated by TGF-b (lncRNA-ATB) both highly expressed in neoplastic thrombus, and correlated with metastases development and shorter survival(69). ATB promotes EMT and invasion by competitive binding and inactivating members of the miR-200 family, promoting survival of the metastatic cells by increasing the stability of IL11 mRNA and activation of STAT3 signaling at metastatic sites(70). High expression of the LncRNA MVIH is associated with microvascular invasion and is an independent risk factor for recurrence following HCC resection(71). Among lncRNAs with tumor suppressive function, CPS1-IT1 and AOC4P showed reduced expression in up to $70 \%$ of HCC samples potentially affecting prognosis through EMT suppression(72,73). The integrated analysis of copy number variations and lncRNAs on resected specimens identified the recurrent deletion of PRAL (p53 regulation-associated lncRNA) to be associated with poor survival in HCC patients. PRAL enhanced p53 stability by facilitating the combination of HSP90 and p53 and therefore competitively inhibiting the p53 negative regulator Mdm2(74).

\section{CircRNA in HCC}

Circular RNA (circRNA) recently emerged as a widespread and abundant class of RNA transcripts that play an active role in gene regulation. CircRNA are generated by back-splicing of pre-RNA with the $3^{\prime}$ and $5^{\prime}$ ends that are covalently closed in a continuous loop. They can contain exons, introns or intergene noncoding regions and they function as "molecular sponges" to sequester miRNAs or interact with specific 
proteins to regulate gene expression. Altered circRNAs have been described in HCC suggesting a role in its development and progression.

A noncoding network driven by the circRNA CircCDYL was recently found to be specifically upregulated in very early stage HCCs. CircCDYL inhibits miR-892a and miR-328-3p expression and activates the PI3K-AKT- mTORC1/ $\beta$-catenin and NOTCH2 pathways, increasing the proportion of stem-like cells within the tumor(75). By using circRNA seq, circASAP1was found overexpressed in resected HCC tissue and plasma of patients experiencing ecurrence in the lungs and poor prognosis. CircASAP1 acts by sponging tumor suppressive miR-326 and miR-532-5p, which results into MAPK signaling activation and increased TAM infiltration(76).

Circular RNA MAT2B (circMAT2B) has a role in reprogramming cancer metabolism by increasing glycolysis under tumoral hypoxia. CircMAT2B upregulates PKM2 activity, a key enzyme in the glycolysis process, by sponging miR-338-3p and blocking PKM2 degradation(77).

Among dowregulated circRNAs, CircMTO1 targets the oncogenic miR-9, thus inhibiting p21 and being associated with shortened survival(78). Although RNA and the protein encoded by SMARCA5 are usually upregulated in HCC, suggesting its potential role as tumor promoter, its circular product, cSMARCA5, is downregulated due to the increased activity of the nuclear RNA helicase DHX9. cSMARCA5 promotes expression of the tumor suppressor TIMP3 by sponging miR-17-3p and miR-181b-5p and its downregulation is associated with aggressive clinicopathological characteristics and poor prognosis(79).

\section{Future perspectives: targeting ncRNAs for HCC treatment}

Targeting ncRNAs represent a promising therapeutic strategy by pursuing inhibition or replacement of miRNAs, splicing regulation via intronic RNAs, and establishment of long ncRNA-based therapeutics to target repetitive RNA and modulation of gene expression. However, despite success of preclinical models, their clinical application remains in its infancy.

The systemic administration of miR-26a in HCC mouse model, using adeno-associated virus as a platform for miRNA delivery, resulted in the inhibition of cancer cell proliferation and reduction of tumor size and nodules(80). Similarly, the systemic delivery of miR-101 in HCC mouse models, using a lentivirus-based vector, resulted in inhibition of tumor angiogenesis and cancer cell invasiveness, suppressing intrahepatic and distant metastases. Both studies did not report significant toxicity in animals(81). However, the delivery of miRNA mimics remain more challenging in humans, with the risk of RNA degradation and inactivation via blood stream. To overcome this issue encapsulation of miRNA in nanovescicles carriers has been recently pursued with success in terms of deliverability. However, two issues are still to be resolved: 1) tissue specificity for delivery to the target-organ, and 2) side effects from the carriers. While delivery to liver is somewhat less challenging than other organs due to the liver double circulation, the replacement of oncosuppressive miRNAs has still found limitation in HCC as well as in other cancers.

This article is protected by copyright. All rights reserved 
Liposomal IV injection of miRRX34, (Mirna Therapeutics), in patients with advanced or metastatic cancer, including unresectable HCC, was evaluated in a phase I clinical trials. However, the trial was terminated due to immune related serious adverse events observed in five patients(82). Consistent with these findings, a phase I trial investigating miRNA replacement in mesothelioma patients confirmed a severe immunological toxicity mediated by nanoparticles, suggesting that better delivery strategies for miRNAs are necessary for human studies. Another limitation of the replacement strategy stems from the introduction of supra-physiological levels of miRNAs, beyond that usually present within cells, inducing over-saturation of the targets and high toxicity. Subsequently, greater attention has been given to inhibitory strategies where the aberrant expression of a given non-coding RNA is counteracted by inhibitory molecules that eliminate the disproportional level without introducing external unphysiological active molecules. Clinical trials in cancers and other diseases are ongoing with Locked Nucleic Acid miRNA inhibitors, which seem to be feasible and less toxic. However, results have to be awaited before proving their efficacy.

\section{Conclusions}

Although recent studies have provided great insights into the importance of ncRNA in enhancing HCC initiation and progression, there remains much to be elucidated. Functional analyses integrated with clinical data have exposed a complex regulatory network in which several classes of ncRNAs interact via a complex network to regulate cell homeostasis and promote malignant phenotypes. NcRNAs primarily exert regulatory role that drives different phenotypes by controlling several intracellular pathways, communication between cancer cells, as well as other cell types in the microenvironment. Therefore, it is likely that a combination of several models that depict intracellular epithelial features (2D cell lines), cellto-cell interaction (3D cancer models), cross-talk between different cell types (co-cultures) and host components including the immune response (in vivo models) are necessary to elucidate the regulatory role of ncRNAs in HCC. Association of pre-clinical investigations with large-scale studies embedded within clinical trials may provide the translational evidence to identify clinically relevant and reproducible noncoding RNAs for clinical use.

This article is protected by copyright. All rights reserved 


\section{REFERENCES}

1. Cancer Genome Atlas Research Network. Electronic address: wheeler@bcm.edu, Cancer Genome Atlas Research Network. Comprehensive and Integrative Genomic Characterization of Hepatocellular Carcinoma. Cell. 2017;169:1327-1341.e23.

2. Delaunay S, Frye M. RNA modifications regulating cell fate in cancer. Nat. Cell Biol. 2019;21:552559.

3. Slack FJ, Chinnaiyan AM. The Role of Non-coding RNAs in Oncology. Cell. 2019;

4. Ha M, Kim VN. Regulation of microRNA biogenesis. Nat. Rev. Mol. Cell Biol. 2014;15:509-524.

5. Luna JM, Barajas JM, Teng K yu, Sun HL, Moore MJ, Rice CM, et al. Argonaute CLIP Defines a Deregulated miR-122-Bound Transcriptome that Correlates with Patient Survival in Human Liver Cancer. Mol. Cell. 2017;

6. Caruso S, Calderaro J, Letouzé E, Nault J-C, Couchy G, Boulai A, et al. Germline and somatic DICER1 mutations in familial and sporadic liver tumors. J. Hepatol. 2017;66:734-742.

7. Lu J, Getz G, Miska EA, Alvarez-Saavedra E, Lamb J, Peck D, et al. MicroRNA expression profiles classify human cancers. Nature. 2005;435:834-8.

8. Esau C, Davis S, Murray SF, Yu XX, Pandey SK, Pear M, et al. miR-122 regulation of lipid metabolism revealed by in vivo antisense targeting. Cell Metab. 2006;3:87-98.

9. Tsai WC, Hsu S Da, Hsu CS, Lai TC, Chen SJ, Shen R, et al. MicroRNA-122 plays a critical role in liver homeostasis and hepatocarcinogenesis. J. Clin. Invest. 2012;122:2884-2897.

10. Ura S, Honda M, Yamashita T, Ueda T, Takatori H, Nishino R, et al. Differential microRNA expression between hepatitis $\mathrm{B}$ and hepatitis $\mathrm{C}$ leading disease progression to hepatocellular carcinoma. Hepatology. 2009;49:1098-1112.

11. Cheung O, Puri P, Eicken C, Contos MJ, Mirshahi F, Maher JW, et al. Nonalcoholic steatohepatitis is associated with altered hepatic MicroRNA expression. Hepatology. 2008;48:1810-1820.

12. Wong CM, Wong CCL, Lee JMF, Fan DNY, Au SLK, Ng IOL. Sequential alterations of microrna expression in hepatocellular carcinoma development and venous metastasis. Hepatology. 2012;55:1453-1461.

13. Valdmanis PN, Kim HK, Chu K, Zhang F, Xu J, Munding EM, et al. miR-122 removal in the liver activates imprinted microRNAs and enables more effective microRNA-mediated gene repression. Nat. Commun. 2018;9:1-9.

14. Gérard C, Di-Luoffo M, Gonay L, Caruso S, Couchy G, Loriot A, et al. Dynamics and predicted drug response of a gene network linking dedifferentiation with beta-catenin dysfunction in hepatocellular carcinoma. J. Hepatol. 2019;71:323-332.

15. Ying Q, Liang L, Guo W, Zha R, Tian Q, Huang S, et al. Hypoxia-inducible MicroRNA-210 
augments the metastatic potential of tumor cells by targeting vacuole membrane protein 1 in hepatocellular carcinoma. Hepatology. 2011;54:2064-2075.

16. Kai AKL, Chan LK, Lo RCL, Lee JMF, Wong CCL, Wong JCM, et al. Down-regulation of TIMP2 by HIF-1 $\alpha /$ miR-210/HIF-3 $\alpha$ regulatory feedback circuit enhances cancer metastasis in hepatocellular carcinoma. Hepatology. 2016;64:473-487.

17. Fang JH, Zhou HC, Zeng C, Yang J, Liu Y, Huang X, et al. MicroRNA-29b suppresses tumor angiogenesis, invasion, and metastasis by regulating matrix metalloproteinase 2 expression. Hepatology. 2011;54:1729-1740.

18. Fang J-H, Zhou H-C, Zhang C, Shang L-R, Zhang L, Xu J, et al. A novel vascular pattern promotes metastasis of hepatocellular carcinoma in an epithelial-mesenchymal transition-independent manner. Hepatology. 2015;62:452-465.

19. Zhou H-C, Fang J-H, Shang L-R, Zhang Z-J, Sang Y, Xu L, et al. MicroRNAs miR-125b and miR100 suppress metastasis of hepatocellular carcinoma by disrupting the formation of vessels that encapsulate tumour clusters. J. Pathol. 2016;240:450-460.

20. Zheng F, Liao Y-J, Cai M-Y, Liu Y-H, Liu T-H, Chen S-P, et al. The putative tumour suppressor microRNA-124 modulates hepatocellular carcinoma cell aggressiveness by repressing ROCK2 and EZH2. Gut. 2012;61:278-289.

21. Wong CC, Wong C, Tung EK, Au SL, Lee JM, Poon RT, et al. The MicroRNA miR-139 suppresses metastasis and progression of hepatocellular carcinoma by down-regulating rho-kinase 2 .

Gastroenterology. 2011;140:322-331.

22. Wong C-M, Wei L, Au SL-K, Fan DN-Y, Zhou Y, Tsang FH-C, et al. MiR-200b/200c/429 subfamily negatively regulates Rho/ROCK signaling pathway to suppress hepatocellular carcinoma metastasis. Oncotarget. 2015;6.

23. Fang T, Lv H, Lv G, Li T, Wang C, Han Q, et al. Tumor-derived exosomal miR-1247-3p induces cancer-associated fibroblast activation to foster lung metastasis of liver cancer. Nat. Commun. 2018;9:1-13.

24. Wang F, Li L, Piontek K, Sakaguchi M, Selaru FM. Exosome miR-335 as a novel therapeutic strategy in hepatocellular carcinoma. Hepatology. 2018;67:940-954.

25. Yang P, Li QJ, Feng Y, Zhang Y, Markowitz GJ, Ning S, et al. TGF- $\beta$-miR-34a-CCL22 SignalingInduced Treg Cell Recruitment Promotes Venous Metastases of HBV-Positive Hepatocellular Carcinoma. Cancer Cell. 2012;22:291-303.

26. Zhou S-L, Hu Z-Q, Zhou Z-J, Dai Z, Wang Z, Cao Y, et al. miR-28-5p-IL-34-macrophage feedback loop modulates hepatocellular carcinoma metastasis. Hepatology. 2016;63:1560-1575. Zhou S, Yin D, Hu Z, Luo C, Zhou Z, Xin H, et al. A Positive Feedback Loop Between Cancer Stem-Like Cells and Tumor-Associated Neutrophils Controls Hepatocellular Carcinoma 
Progression. Hepatology. 2019;70:1214-1230.

28. Murakami Y, Yasuda T, Saigo K, Urashima T, Toyoda H, Okanoue T, et al. Comprehensive analysis of microRNA expression patterns in hepatocellular carcinoma and non-tumorous tissues. Oncogene. 2006;25:2537-2545.

29. Pineau P, Volinia S, McJunkin K, Marchio A, Battiston C, Terris B, et al. miR-221 overexpression contributes to liver tumorigenesis. Proc. Natl. Acad. Sci. U. S. A. 2010;107:264-269.

30. Arroyo JD, Chevillet JR, Kroh EM, Ruf IK, Pritchard CC, Gibson DF, et al. Argonaute2 complexes carry a population of circulating microRNAs independent of vesicles in human plasma. Proc. Natl. Acad. Sci. U. S. A. 2011;108:5003-5008.

31. Li L-M, Hu Z-B, Zhou Z-X, Chen X, Liu F-Y, Zhang J-F, et al. Serum microRNA Profiles Serve as Novel Biomarkers for HBV Infection and Diagnosis of HBV-Positive Hepatocarcinoma. Cancer Res. 2010;70:9798-9807.

32. Zhou J, Yu L, Gao X, Hu J, Wang J, Dai Z, et al. Plasma MicroRNA Panel to Diagnose Hepatitis B Virus-Related Hepatocellular Carcinoma. J. Clin. Oncol. 2011;29:4781-4788.

33. Zhang Y, Dai J, Deng H, Wan H, Liu M, Wang J, et al. miR-1228 promotes the proliferation and metastasis of hepatoma cells through a p53 forward feedback loop. Br. J. Cancer. 2015;112:365374.

34. Lin X-J, Chong Y, Guo Z-W, Xie C, Yang X-J, Zhang Q, et al. A serum microRNA classifier for early detection of hepatocellular carcinoma: a multicentre, retrospective, longitudinal biomarker identification study with a nested case-control study. Lancet Oncol. 2015;16:804-815.

35. Ji J, Shi J, Budhu A, Yu Z, Forgues M, Roessler S, et al. MicroRNA expression, survival, and response to interferon in liver cancer. N. Engl. J. Med. 2009;361:1437-1447.

36. Fu Q, Yang F, Xiang T, Huai G, Yang X, Wei L, et al. A novel microRNA signature predicts survival in liver hepatocellular carcinoma after hepatectomy. Sci. Rep. 2018;

37. Zhen Y, Xinghui Z, Chao W, Yi Z, Jinwen C, Ruifang G, et al. Several microRNAs could predict survival in patients with hepatitis B-related liver cancer. Sci. Rep. 2017;7:1-8.

38. Braconi C, Valeri N, Gasparini P, Huang N, Taccioli C, Nuovo G, et al. Hepatitis C virus proteins modulate microRNA expression and chemosensitivity in malignant hepatocytes. Clin. Cancer Res. 2010;

39. Caruso S, Calatayud A-L, Pilet J, La Bella T, Rekik S, Imbeaud S, et al. Analysis of Liver Cancer Cell Lines Identifies Agents With Likely Efficacy Against Hepatocellular Carcinoma and Markers of Response. Gastroenterology. 2019;157:760-776.

40. Fornari F, Pollutri D, Patrizi C, La Bella T, Marinelli S, Casadei Gardini A, et al. In hepatocellular carcinoma miR-221 modulates sorafenib resistance through inhibition of caspase-3-mediated apoptosis. Clin. Cancer Res. 2017;23:3953-3965.

This article is protected by copyright. All rights reserved 
41. Nishida N, Arizumi T, Hagiwara S, Ida H, Sakurai T, Kudo M. MicroRNAs for the Prediction of Early Response to Sorafenib Treatment in Human Hepatocellular Carcinoma. Liver Cancer. 2017;

42. Bruix J, Qin S, Merle P, Granito A, Huang YH, Bodoky G, et al. Regorafenib for patients with hepatocellular carcinoma who progressed on sorafenib treatment (RESORCE): a randomised, double-blind, placebo-controlled, phase 3 trial. Lancet. 2017;389:56-66.

43. Teufel M, Seidel H, Köchert K, Meinhardt G, Finn RS, Llovet JM, et al. Biomarkers Associated With Response to Regorafenib in Patients With Hepatocellular Carcinoma. Gastroenterology. 2019;156:1731-1741.

44. Finn RS, Chan SL, Zhu AX, Knox JJ, Cheng A-L, Siegel AB, et al. KEYNOTE-240: Randomized phase III study of pembrolizumab versus best supportive care for second-line advanced hepatocellular carcinoma. J. Clin. Oncol. 2017;35:TPS503-TPS503.

45. Yau T, Park JW, Finn RS, Cheng A-L, Mathurin P, Edeline J, et al. LBA38_PRCheckMate 459: A randomized, multi-center phase III study of nivolumab (NIVO) vs sorafenib (SOR) as first-line (1L) treatment in patients (pts) with advanced hepatocellular carcinoma (aHCC). Ann. Oncol. 2019;

46. Boeri M, Milione M, Proto C, Signorelli D, Russo G Lo, Galeone C, et al. Circulating miRNAs and PD-L1 tumor expression are associated with survival in advanced NSCLC patients treated with immunotherapy: A prospective study. Clin. Cancer Res. 2019;

47. Huber V, Vallacchi V, Fleming V, Hu X, Cova A, Dugo M, et al. Tumor-derived microRNAs induce myeloid suppressor cells and predict immunotherapy resistance in melanoma. J. Clin. Invest. 2018;128:5505-5516.

48. Ranzani V, Rossetti G, Panzeri I, Arrigoni A, Bonnal RJP, Curti S, et al. The long intergenic noncoding RNA landscape of human lymphocytes highlights the regulation of $\mathrm{T}$ cell differentiation by linc-MAF-4. Nat. Immunol. 2015;

49. Tanne A, Muniz LR, Puzio-Kuter A, Leonova KI, Gudkov A V., Ting DT, et al. Distinguishing the immunostimulatory properties of noncoding RNAs expressed in cancer cells. Proc. Natl. Acad. Sci. U. S. A. 2015;112:15154-15159.

50. Cabili M, Trapnell C, Goff L, Koziol M, Tazon-Vega B, Regev A, et al. Integrative annotation of human large intergenic noncoding RNAs reveals global properties and specific subclasses. Genes Dev. 2011;

51. Salmena L, Poliseno L, Tay Y, Kats L, Pandolfi PP. A ceRNA Hypothesis: The Rosetta Stone of a Hidden RNA Language? Cell. 2011;146:353-358.

52. Fu WM, Zhu X, Wang WM, Lu YF, Hu BG, Wang H, et al. Hotair mediates hepatocarcinogenesis through suppressing miRNA-218 expression and activating P14 and P16 signaling. J. Hepatol. 2015;63:886-895.

53. Yang F, Zhang L, Huo X, Yuan J, Xu D, Yuan S, et al. Long noncoding RNA high expression in 
hepatocellular carcinoma facilitates tumor growth through enhancer of zeste homolog 2 in humans. Hepatology. 2011;54:1679-1689.

54. Hu J-J, Song W, Zhang S-D, Shen X-H, Qiu X-M, Wu H-Z, et al. HBx-upregulated lncRNA UCA1 promotes cell growth and tumorigenesis by recruiting EZH2 and repressing p27Kip1/CDK2 signaling. Sci. Rep.. 2016;6:23521.

55. Wang F, Ying H-Q, He B-S, Pan Y-Q, Deng Q-W, Sun H-L, et al. Upregulated lncRNA-UCA1 contributes to progression of hepatocellular carcinoma through inhibition of miR-216b and activation of FGFR1/ERK signaling pathway. Oncotarget. 2015;6.

56. Lau C-C, Sun T, Ching AKK, He M, Li J-W, Wong AM, et al. Viral-Human Chimeric Transcript Predisposes Risk to Liver Cancer Development and Progression. Cancer Cell. 2014;25:335-349.

57. Liang H-W, Wang N, Wang Y, Wang F, Fu Z, Yan X, et al. Hepatitis B virus-human chimeric transcript HBx-LINE1 promotes hepatic injury via sequestering cellular microRNA-122. J. Hepatol. 2016;64:278-291.

58. Huang J, Guo Y, Zhao C, Yuan S, Wang Y, Tang G, et al. Hepatitis B virus X protein (HBx)-related long noncoding RNA (lncRNA) down-regulated expression by HBx (Dreh) inhibits hepatocellular carcinoma metastasis by targeting the intermediate filament protein vimentin. Hepatology. 2013;57:1882-1892.

59. Zhang H, Zhu C, Zhao Y, Li M, Wu L, Yang X, et al. Long non-coding RNA expression profiles of hepatitis C virusrelated dysplasia and hepatocellular carcinoma. Oncotarget. 2015;6:43770-43778.

60. Yuan S, Wang J, Yang F, Tao Q, Zhang J, Wang L, et al. Long noncoding RNA DANCR increases stemness features of hepatocellular carcinoma by derepression of CTNNB1. Hepatology. 2016;63:499-511.

61. Cao C, Sun J, Zhang D, Guo X, Xie L, Li X, et al. The long intergenic noncoding RNA UFC1, a target of microRNA 34a, interacts with the mRNA stabilizing protein HuR to increase levels of $\beta$ catenin in HCC cells. Gastroenterology. 2015;148:415-426.e18.

62. Carotenuto P, Fassan M, Pandolfo R, Lampis A, Vicentini C, Cascione L, et al. Wnt signalling modulates transcribed-ultraconserved regions in hepatobiliary cancers. Gut. 2017;

63. Li D, Liu X, Zhou J, Hu J, Zhang D, Liu J, et al. Long noncoding RNA HULC modulates the phosphorylation of YB-1 through serving as a scaffold of extracellular signal-regulated kinase and YB-1 to enhance hepatocarcinogenesis. Hepatology. 2017;65:1612-1627.

64. Li S-P, Xu H-X, Yu Y, He J-D, Wang Z, Xu Y-J, et al. LncRNA HULC enhances epithelialmesenchymal transition to promote tumorigenesis and metastasis of hepatocellular carcinoma via the miR-200a-3p/ZEB1 signaling pathway. Oncotarget. 2016;7.

65. Lu Z, Xiao Z, Liu F, Cui M, Li W, Yang Z, et al. Long non-coding RNA HULC promotes tumor angiogenesis in liver cancer by up-regulating sphingosine kinase 1 (SPHK1). Oncotarget.

This article is protected by copyright. All rights reserved 
$2016 ; 7: 241-254$.

66. Cui M, Xiao Z, Wang Y, Zheng M, Song T, Cai X, et al. Long noncoding RNA HULC modulates abnormal lipid metabolism in hepatoma cells through an mir-9-mediated RXRA signaling pathway. Cancer Res. 2015;75:846-857.

67. Li T, Xie J, Shen C, Cheng D, Shi Y, Wu Z, et al. Upregulation of long noncoding RNA ZEB1-AS1 promotes tumor metastasis and predicts poor prognosis in hepatocellular carcinoma. Oncogene. 2016;35:1575-1584.

68. Li T, Xie J, Shen C, Cheng D, Shi Y, Wu Z, et al. Amplification of Long Noncoding RNA ZFAS1 Promotes Metastasis in Hepatocellular Carcinoma. Cancer Res. 2015;75:3181-3191.

69. Guo W, Liu S, Cheng Y, Lu L, Shi J, Xu G, et al. ICAM-1-related noncoding RNA in cancer stem cells maintains ICAM-1 expression in hepatocellular carcinoma. Clin. Cancer Res. 2016;22:20412050.

70. Yuan J, Yang F, Wang F, Ma J, Guo Y, Tao Q, et al. A Long Noncoding RNA Activated by TGF- $\beta$ Promotes the Invasion-Metastasis Cascade in Hepatocellular Carcinoma. Cancer Cell. 2014;25:666681.

71. Yuan S-X, Yang F, Yang Y, Tao Q-F, Zhang J, Huang G, et al. Long noncoding RNA associated with microvascular invasion in hepatocellular carcinoma promotes angiogenesis and serves as a predictor for hepatocellular carcinoma patients' poor recurrence-free survival after hepatectomy. Hepatology. 2012;56:2231-2241.

72. Wang TH, Yu CC, Lin YS, Chen TC, Yeh CT, Liang KH, et al. Long noncoding RNA CPS1-IT1 suppresses the metastasis of hepatocellular carcinoma by regulating HIF-1 $\beta$ activity and inhibiting epithelial-mesenchymal transition. Oncotarget. 2016;7:43588-43603.

73. Wang TH, Lin YS, Chen Y, Yeh CT, Huang Y lin, Hsieh TH, et al. Long non-coding RNA AOC4P suppresses hepatocellular carcinoma metastasis by enhancing vimentin degradation and inhibiting epithelial-mesenchymal transition. Oncotarget. 2015;6:23342-23357.

74. Zhou C, Yang F, Yuan S, Ma J, Liu F, Yuan J, et al. Systemic genome screening identifies the outcome associated focal loss of long noncoding RNA PRAL in hepatocellular carcinoma.

Hepatology. 2016;63:850-863.

75. Wei Y, Chen X, Liang C, Ling Y, Yang X, Ye X, et al. A Noncoding Regulatory RNAs Network Driven by Circ-CDYL Acts Specifically in the Early Stages Hepatocellular Carcinoma. Hepatology. 2019;0:hep.30795.

76. Hu Z-Q, Zhou S, Li J, Zhou Z, Wang P-C, Xin H-Y, et al. Circular RNA Sequencing Identifies CircASAP1 as a Key Regulator in Hepatocellular Carcinoma Metastasis. Hepatology. 2019;hep.31068.

77. Li Q, Pan X, Zhu D, Deng Z, Jiang R, Wang X. Circular RNA MAT2B Promotes Glycolysis and 
Malignancy of Hepatocellular Carcinoma Through the miR-338-3p/PKM2 Axis Under Hypoxic Stress. Hepatology. 2019;70:1298-1316.

78. Han D, Li J, Wang H, Su X, Hou J, Gu Y, et al. Circular RNA circMTO1 acts as the sponge of microRNA-9 to suppress hepatocellular carcinoma progression. Hepatology. 2017;66:1151-1164.

79. Yu J, Xu Q guo, Wang Z guang, Yang Y, Zhang L, Ma J zhao, et al. Circular RNA cSMARCA5 inhibits growth and metastasis in hepatocellular carcinoma. J. Hepatol. 2018;68:1214-1227.

80. Kota J, Chivukula RR, O’Donnell KA, Wentzel EA, Montgomery CL, Hwang HW, et al. Therapeutic microRNA Delivery Suppresses Tumorigenesis in a Murine Liver Cancer Model. Cell. 2009;137:1005-1017.

81. Zheng F, Liao YJ, Cai MY, Liu TH, Chen SP, Wu PH, et al. Systemic Delivery of MicroRNA-101 Potently Inhibits Hepatocellular Carcinoma In Vivo by Repressing Multiple Targets. PLoS Genet. 2015;11:1-21.

82. https://clinicaltrials.gov/ct2/show/NCT01829971. 2016;

\section{FIGURE LEGENDS}

Figure 1. Biological role of miRNAs in HCC initiation and progression: MiRNAs have an active role in hepatocarcinogenesis depending on the different pathogenic factors and etiologies involved. MiRNAs promote $\mathrm{HCC}$ initiation by enhancing persistent inflammation and by deregulating signaling pathways that control cell cycle and apoptosis. MiRNAs contribute to HCC progression by promoting epithelial to mesenchymal transition (EMT), matrix reorganization and neoangiogenesis and by impairing immunogenicity within tumor microenvironment via the interactions between cancer cells and immune/inflammatory cells, such as cancer associated fibroblasts (CAF), regulatory $\mathrm{T}$ cells (regTcells), tumor associate macrophages (TAM) and tumor associate neutrophils (TAN).

Figure 2. Clinical roles of ncRNAs: Diagnostic: combinations of circulating miRNAs have been proposed to enhance diagnostic accuracy for HCC. Prognostic: Several aberrantly expressed ncRNA in resected specimens have a key role in the development of metastases and can potentially serve as prognostic biomarkers. Predictive: In patients with advanced HCC, circulating miRNAs are associated with the development of drug resistance and disease control in course of Sorafenib. By analysing patients included in the RESORCE trial, a set of miRNAs identify survival benefits in course of Regorafenib.

This article is protected by copyright. All rights reserved 


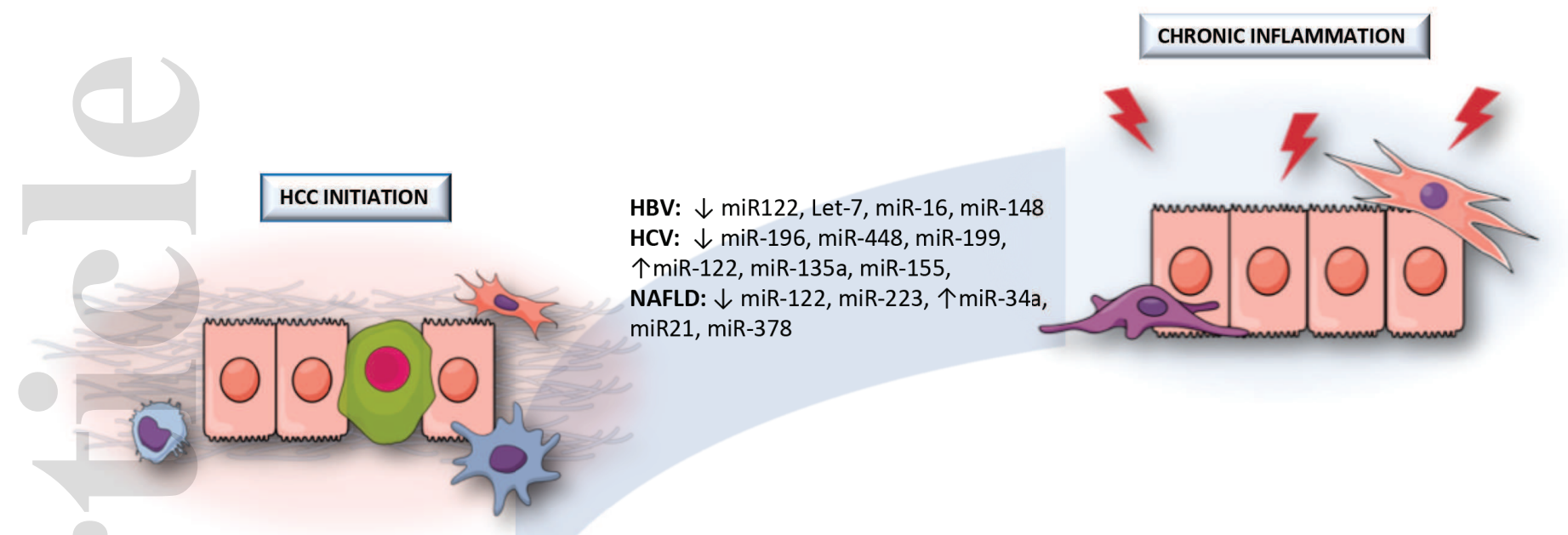

Cell survival and proliferation: $\downarrow$ miR-122, miR-

155, miR-223, miR-193, miR-221, miR-29, Let-7, miR101, miR148, 个 miR-21
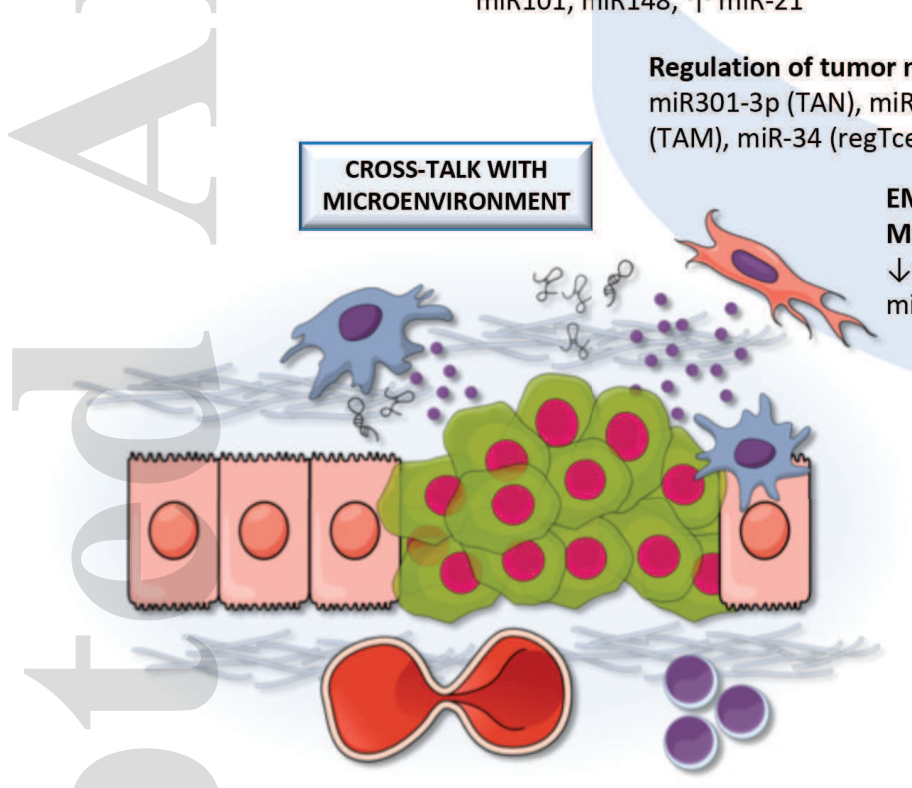

hep_31225_f1.eps
Regulation of tumor microenvironment: $\downarrow$ miR-335 (CAF), miR301-3p (TAN), miR-26, 个 miR1247-3p (CAF), miR-28-5-p (TAM), miR-34 (regTcell)

EMT: $\downarrow$ miR122, miR124, miR139, miR200 Matrix reorganization and angiogenesis: $\downarrow$ miR29, miR125b, miR100, miR101 个 miR-210

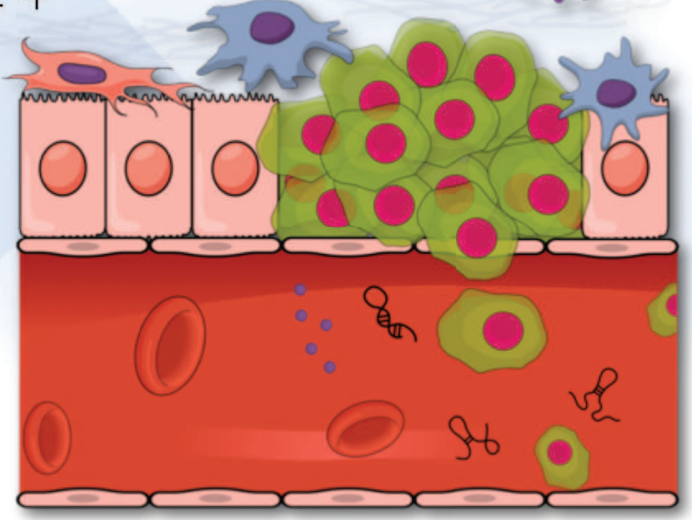


